\title{
CORRECTION
}

\section{Correction to: Position-based modeling of lesion displacement in ultrasound-guided breast biopsy}

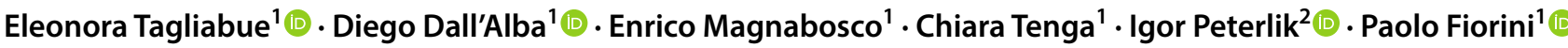

Published online: 27 June 2019

(c) CARS 2019

\section{Correction to: \\ International Journal of Computer Assisted Radiology and Surgery \\ https://doi.org/10.1007/s11548-019-01997-z}

The original version of this article unfortunately contained a mistake. The presentation of Table 2 was incorrect.

The corrected Table 2 is given below.

The original article has been corrected.

Table 2 Optimal values of cluster spacing, radius and stiffness parameters estimated with the proposed optimization strategies for the calibration and breast phantoms. Last columns report the mean error and standard deviation over all the deformations in $\mathrm{mm}$, when each set of parameters is used to predict the position of the landmark used for the fine-tuning process

\begin{tabular}{l|ccc|cc}
\hline & Cluster spacing & Cluster radius & Cluster stiffness & Mean Error & STD \\
\hline Calibration phantom & 9.6001 & 9.1674 & 0.452390 & 6.64 & 2.00 \\
\hline Breast phantom & 11.1626 & 8.5424 & 0.464890 & 5.07 & 1.62 \\
\hline
\end{tabular}

The original article can be found online at https://doi.org/10.1007/ s11548-019-01997-z.

Eleonora Tagliabue

eleonora.tagliabue@univr.it

Paolo Fiorini

paolo.fiorini@univr.it

1 Department of Computer Science, University of Verona, Str.

le Grazie 15, Verona, Italy

2 INRIA, Strasbourg, France
Publisher's Note Springer Nature remains neutral with regard to jurisdictionalclaims in published maps and institutional affiliations. 